\title{
Colorimetric Determination of Ascorbic Acid Based on Its Interfering Effect in the Enzymatic Analysis of Glucose: An Approach Using Smartphone Image Analysis
}

\author{
Mayra S. Coutinho, Camilo L. M. Morais, Ana C. O. Neves, Fabrício G. Menezes and \\ Kássio M. G. Lima* \\ Química Biológica e Quimiometria, Instituto de Química, \\ Universidade Federal do Rio Grande do Norte, 59072-970 Natal-RN, Brazil
}

\begin{abstract}
The use of digital image analysis as an analytical tool is a reality nowadays, and the use of smartphones stands out due to its high accessibility and practicality. Ascorbic acid (AA) is a natural and essential vitamin available as a supplement as a result of its use in preventing and treating several pathologies. This paper reports a simple, fast and low cost method using smartphone image analysis for quantification of AA based on its interfering effect in the enzymatic colorimetric detection of glucose. Commercial vitamin $\mathrm{C}$ tablets were used as prediction set for AA quantification, showing very satisfactory results (relative errors $<4 \%$ ), where no statistical difference at a $95 \%$ confidence level was observed between the AA content estimated by the imaging method and the labeled reference values. As advantages, this method does not use expensive reagents neither laborious procedures to carry out the analysis.
\end{abstract}

Keywords: ascorbic acid, glucose analysis interfering, enzymatic colorimetric method, image analysis

\section{Introduction}

The development of new analytical methodologies based on digital imaging has been attracting considerable attention in the last decade due to its low cost, simplicity, non-destructive aspect and speed of analysis. Common digital image capturing devices such as cameras, webcams, scanners, and smartphones are used to register images from colorimetric reactions, in which the intensity of the developed color is directly proportional to the analyte concentration. To demonstrate these features, several papers involving colorimetric detection have been published such as for the quantification of synthetic dyes; ${ }^{1}$ monitoring of organic reactions; $;^{1,2}$ evaluation of food quality; ${ }^{3}$ characterization of drug authenticity; ${ }^{4}$ and determination of biochemical parameters. ${ }^{5-9}$ Among several devices applied in image-based analytical methodologies, the use of smartphones has emerged as a notable tool due to the massive amount of users all over the world, easy image acquisition and high accessibility for data transmission. ${ }^{4-8}$

Ascorbic acid (AA) is a natural water-soluble vitamin widespread in nature that plays a crucial role in biological processes and metabolism, ${ }^{10} \mathrm{AA}$ is a common constituent

*e-mail: kassiolima@gmail.com of the human diet and commercialized as a supplement (vitamin C) in high levels, since its ingestion is strongly suggested for prevention and treatment of several pathologies.

Several approaches have been reported in an attempt to determine AA levels in different matrices, such as the high-performance liquid chromatography (HPLC) and ultra performance liquid chromatography (UPLC) methods, electrochemical measurements, as well as molecular spectroscopy such as UV-Vis and fluorescence. ${ }^{11-18}$ These methods can be very attractive in terms of limit of detection (LOD) and quantification (LOQ), which can include values around 0.3 and $1.0 \mu \mathrm{g} \mathrm{dL} \mathrm{d}^{-1}$, respectively, making analysis of samples containing very low concentrations levels possible. ${ }^{11-15}$ However, they present some considerable drawbacks, such as the dependence on sophisticated instruments; time-consuming analysis; need for expensive reagents in high volumes; and have laborious procedures. In addition to these reported methods for quantification of AA, there are some commercially available assays, usually based in enzymatic reagents presented in very specific medium, involving colorimetric/ fluorometric spectroscopies, and although they allow quantification in low levels, they are very expensive, which may limit their use for some routine applications. 
The advantages of the recent methods based in image analysis consist in overcoming at least some of these drawbacks. In the literature, there are only a few works which have reported the quantification of AA in solution by image analysis. ${ }^{17-20}$ In fact, these methods are very interesting since they involve modern aspects of chemistry, such as nanoparticle formations and click reactions. On the other hand, some drawbacks remain such as the need for expensive reagents, laborious procedures and being timeconsuming. Also, the limits of quantification are not as low as conventional analysis; however, this does not exclude the relevance of the method since some fruits and commercial supplements are composed of high levels of AA.

Therefore, in this paper we present a new method based on digital images acquired with a smartphone for determining AA in aqueous solution. For this purpose, we take advantage of the effect that AA causes in the analysis of glucose through the gold standard spectroscopic-based enzymatic colorimetric method (ECM) ${ }^{21}$ More specifically, AA acts on suppressing the formation of quinoneimine dye (essential for UV-Vis analysis), which induces lower color intensity of a glucose solution as the concentration of AA increases. The results suggest that it is possible to apply the method herein described in the preliminary analysis of commercial vitamin C. Although the drawback originated by the presence of AA in samples subjected to glucose analysis has been known for decades, to the best of our knowledge, herein we report the first time that this interference effect was measured and associated to quantitative purposes.

\section{Experimental}

\section{Reagents and instrumentation}

L-Ascorbic acid and D-glucose were both acquired from Sigma-Aldrich and used without further purification. Glucose mono reagent (color reagent) kit was acquired from Bioclin (Bioclin Quibasa, Brazil) and stored as instructed by the manufacturer. This color reagent is composed of a buffer ( $\mathrm{pH}$ 7.0); phenol (10 mmol L-1); 4-aminoantipyrine $\left(0.3 \mathrm{mmol} \mathrm{L}^{-1}\right)$; sodium azide $\left(7.7 \mathrm{mmol} \mathrm{L}^{-1}\right)$; glucose oxidase (>10.000 UI); and peroxidase (> $700 \mathrm{UI})$. All experiments were carried out in deionized water. Commercial vitamin C tablets were purchased in drugstores. A Hemoquímica model HM0064D water-bath was used in the experiments. UV-Vis analyses were carried out in an Evolution 60S model Thermo Scientific spectrophotometer using $1 \mathrm{~cm}$ length quartz cuvettes. All images were acquired by using a Sony Xperia C2304 smartphone with camera resolution of 8 megapixels.

\section{Samples}

The calibration set was built from five calibration samples in triplicate containing $10.0 \mathrm{mg} \mathrm{L}^{-1}$ of glucose and different amounts of AA $\left(6.0,7.0,8.0,9.0\right.$ and $\left.10.0 \mathrm{mg} \mathrm{L}^{-1}\right)$. All solutions were prepared in deionized water, which is very important since AA undergoes oxidation in the presence of metal, such as copper. On the other hand, in the absence of any catalyst (such as copper or hydrogen peroxide), the presence of dissolved oxygen is usually insignificant in the oxidation of $\mathrm{AA} .{ }^{22}$ All solutions were prepared just before use. Analysis of vitamin $\mathrm{C}$ in commercial tablets (brands A-C) was performed by dissolving their content in water until concentration of $8.0 \mathrm{mg} \mathrm{L}^{-1}$.

\section{UV-Vis spectroscopy}

UV-Vis analysis was performed as a reference method for the quantification of AA through image analysis. UV-Vis spectra were acquired for the calibration samples by adding $20 \mu \mathrm{L}$ of the solutions containing D-glucose (10.0 $\mathrm{mg} \mathrm{L}^{-1}$ ) and L-ascorbic acid (varying from 6.0 to $10.0 \mathrm{mg} \mathrm{L}^{-1}$ ) to $2 \mathrm{~mL}$ of the color reagent after heating at $37{ }^{\circ} \mathrm{C}$ for $10 \mathrm{~min}$. The blank was the color reagent for all cases. Changes in the absorbance were analyzed at $508 \mathrm{~nm}$ of the UV-Vis spectra.

\section{Image acquisition}

The images from the same samples measured by UV-Vis spectroscopy were also captured by using a smartphone. The same cuvettes analyzed by the spectrometer were placed onto a white surface with a white background and the pictures were taken with the smartphone held at a statistic position of $19.5 \mathrm{~cm}$ from the bucket, avoiding blurriness. In addition, blank images (images of cuvettes filled with deionized water) were also recorded in order to avoid ambient lighting interference and further used to obtain the RGB (red-green-blue) absorbance. The spectra and images were acquired immediately after the samples were prepared. This experimental setup is illustrated in Figure 1a.

After acquiring the sample images in .JPEG format with $96 \times 96$ dpi resolution, they were loaded into a personal computer to extract the region of interest (ROI) composed of a square of $10 \times 10$ pixels (Figure $1 \mathrm{~b}$ ). This procedure was done using GIMP 2.0 software..$^{23}$

\section{Digital image analysis}

The ROI of the images (Figure 1b) were processed 
a.

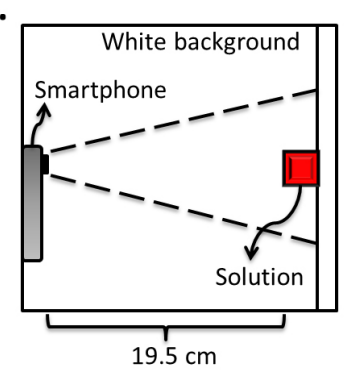

b.

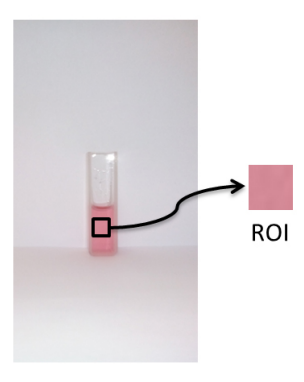

Figure 1. (a) Illustration of experimental setup for image acquisition; (b) example of region of interest (ROI) cropped from a sample image of AA.

using MATLAB R2014a environment (Math Works, USA) through the calculation of the RGB-resolved absorbance as the analytical signal univariately related to the concentration of AA, according to equation $1::^{24}$

$A_{k}=-\log \frac{I_{k}}{I_{0, k}}$

where, $A_{k}$ is the RGB-resolved absorbance for a given color channel $\mathrm{k}$ (red, green or blue); $\mathrm{I}_{\mathrm{k}}$ is the intensity for a given color channel $\mathrm{k}$; and $\mathrm{I}_{0, \mathrm{k}}$ is the intensity of blank for a given color channel $\mathrm{k}$. The intensity value is calculated as the mean for its related color channel.

\section{Statistical evaluation}

In this study, the performance of the calibration model was evaluated by comparing the AA predicted values by the imaging method with the AA measured values. This was made by calculating the statistic RMSEC (root mean square error of calibration), relative standard-deviation (RSD), bias (average difference between predicted and measured values), relative error, accuracy, R (correlation coefficient), $\mathrm{R}^{2}$ (coefficient of determination), limit of detection (LOD) and limit of quantification (LOQ). Additionally, the Student's $t$-test was performed to find any significant difference in the results of RGB prediction and AA measurements. The LOD and LOQ were calculated as follows:

$\mathrm{LOD}=\frac{3.3 \mathrm{~S}_{\mathrm{y} / \mathrm{x}}}{\mathrm{A}} \sqrt{1+\mathrm{h}_{0}+\frac{1}{\mathrm{I}}}$

$\mathrm{LOQ}=\frac{10 \mathrm{~S}_{\mathrm{y} / \mathrm{x}}}{\mathrm{A}} \sqrt{1+\mathrm{h}_{0}+\frac{1}{\mathrm{I}}}$

where, $S_{y / x}$ is the residual standard deviation; $A$ is the slope of the univariate calibration curve; $h_{0}$ is the leverage for the blank sample; and I is the number of calibration samples. The leverage for the blank sample $\mathrm{h}_{0}$ can be estimated as: ${ }^{25}$

$$
\mathrm{h}_{0}=\frac{\overline{\mathrm{c}}_{\mathrm{cal}}^{2}}{\sum_{\mathrm{i}=1}^{\mathrm{I}}\left(\mathrm{c}_{\mathrm{i}}-\overline{\mathrm{c}}_{\mathrm{cal}}\right)^{2}}
$$

where, $\bar{c}_{\text {cal }}$ is the mean calibration concentration and $\mathrm{c}_{\mathrm{i}}$ is each of the calibration concentration values.

\section{Results and Discussion}

In the ECM method for quantification of glucose, the referred carbohydrate (as $(\alpha+\beta)$-D-glucopyranose) is oxidized to $\delta$-gluconolactone by molecular oxygen in the presence of enzyme glucose oxidase. Sequentially, hydrogen peroxide, which is also generated in this first stage, induces oxidative condensation of 4-aminoantipyrine and phenol by the action of enzyme peroxidase to afford a quinoneimine dye (Figure 2a). ${ }^{21}$ Since both glucose oxidation and synthetic dye formation in the ECM are based on oxidative processes, it is possible to assume that AA can compete with the substrates present in the reaction media and be previously oxidized to the dye formation to afford dehydroascorbic acid (DHA, Figure 2a, inset). Colors of the solutions containing available enzymatic reagent with a specified amount of glucose $\left(10.0 \mathrm{mg} \mathrm{L}^{-1}\right)$ will become less intense as the concentration of AA increases in the solution, and this effect may be followed by both UV-Vis spectroscopy and image analysis (Figure 2b).

a.

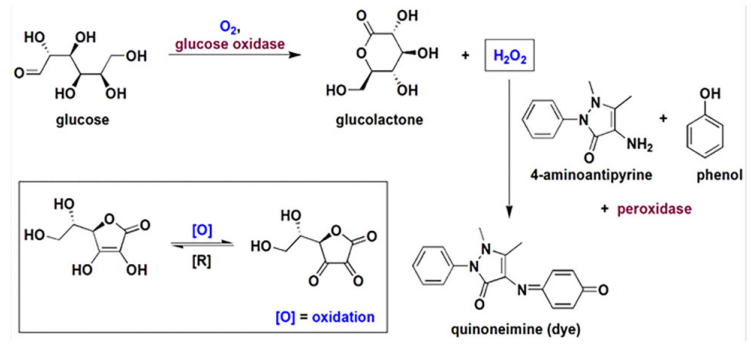

b.

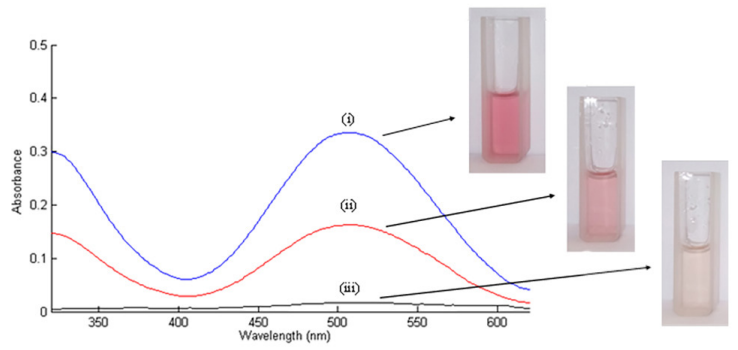

Figure 2. (a) Reactions involved in the formation of quinoneimine from initial oxidation of glucose and subsequent condensation of 4-aminoantipyrine and phenol. Inset: oxidation of AA into dehydroascorbic acid in the reaction media; (b) changes in UV-Vis spectra (320-650 nm) and color intensity of the solutions of colorimetric reagent containing $20 \mu \mathrm{L}$ of glucose solution $\left(10.0 \mathrm{mg} \mathrm{L}^{-1}\right)$ in $2 \mathrm{~mL}$ of color reagent upon addition of AA: (i) $0 \mathrm{mg} \mathrm{L}^{-1}$; (ii) $5.0 \mathrm{mg} \mathrm{L}^{-1}$; (iii) $10.0 \mathrm{mg} \mathrm{L}^{-1}$. 


\section{Calibration set using standard ascorbic acid}

Since the gold standard ECM for determination of glucose is based in UV-Vis spectroscopy measurements, we started our studies by evaluating the changes in the UV-Vis spectra of the enzymatic color reagent after adding different amounts of AA (6.0-10.0 $\left.\mathrm{mg} \mathrm{L}^{-1}\right)$ in solutions containing $10.0 \mathrm{mg} \mathrm{L}^{-1}$ of glucose. A calibration curve $(\mathrm{y}=-0.0310 \mathrm{x}+0.336)$ was found with an $\mathrm{R}^{2}$ equal to 0.998 (Figure 3a) for UV-Vis data, presenting an RMSEC of $0.06 \mathrm{mg} \mathrm{L}^{-1}$ and a relative error for calibration of $0.76 \%$. After UV-Vis measurements, the images captured with the smartphone were analyzed and the same trend was observed using this method, where the G-B (green-blue) resolved absorbance values were used to build the calibration curve $(\mathrm{y}=-0.00526 \mathrm{x}+0.0560)$ with an $\mathrm{R}^{2}$ equal to 0.972 (Figure $3 \mathrm{~b}$ ). The $\mathrm{G}-\mathrm{B}$ resolved absorbance represents the region between 546.1 and $435.8 \mathrm{~nm}$, covering the region of the absorption band for AA chromogen $\left(\lambda_{\max }=508 \mathrm{~nm}\right)$. The RMSEC found using the image method was equal to $0.24 \mathrm{mg} \mathrm{L}^{-1}$ and a relative error for calibration of $2.91 \%$ was found.

Although the UV-Vis method presented better sensitivity and smaller error, the relative error found in the calibration set using the image method was significantly small $(<3 \%)$, indicating good accuracy using the synthetic samples. The linearity of the curves were also evaluated according to the statistical $F$ test. ${ }^{25,26}$ For both techniques, the calculated $F$ values $(0.050$ and 0.164 for UV-Vis and image method, respectively) were smaller than the critical $F$ value $(6.39, \alpha=0.05)$ for a confidence level of $95 \%$, proving the linearity of the calibration curves. In addition, the low values of bias found for both methods indicate absence of systematic error. The figures of merit for the methods using both UV-Vis and image-based approaches are shown in Table 1.

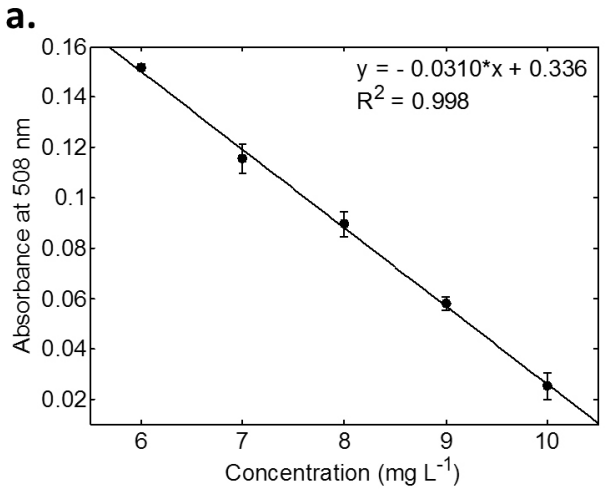

Table 1. Figures of merit for the calibration set based on UV-Vis spectroscopy and image method

\begin{tabular}{lcc}
\hline Figure of merit & UV-Vis method & Image method \\
\hline RMSEC / (mg L-1) & 0.06 & 0.24 \\
Bias / (mg L $\left.{ }^{-1}\right)$ & 0.002 & -0.006 \\
Relative error / \% & 0.76 & 2.91 \\
R & 0.999 & 0.986 \\
$\mathrm{R}^{2}$ & 0.998 & 0.972 \\
Sensitivity & 0.0310 & 0.00526 \\
\hline
\end{tabular}

RMSEC: root mean square error of calibration; R: correlation coefficient; $\mathrm{R}^{2}$ : coefficient of determination.

Image analysis applied to commercial vitamin C tablets

After confirming a linear trend found as a consequence of AA in the formation of quinoneimine dye, we then aimed to validate this methodology. Firstly, an external validation test was performed using three synthetic samples with $7.5 \mathrm{mg} \mathrm{L}^{-1}$ of ascorbic acid. Validation response of $7.7 \pm 0.1 \mathrm{mg} \mathrm{L}^{-1}$ (relative error of $2.67 \%$ ) and $7.5 \pm 0.3 \mathrm{mg} \mathrm{L}^{-1}$ (relative error of $0.04 \%$ ) was found for UV-Vis and image method, respectively. These results confirmed the ability of the proposed method using images to predict test samples with low error. Thereafter, commercial effervescent vitamin $\mathrm{C}$ samples (brands A-C) were analyzed in order to evaluate the proposed method in real environment. The nominal and predicted AA concentrations for these commercial samples are shown in Table 2.

As shown in Table 2, the nominal and predicted concentrations of AA in commercial vitamin $\mathrm{C}$ samples were very similar using the imaging method, with relative errors smaller than $4 \%$. These relative errors were a little higher than using UV-Vis spectroscopy, which could be caused due to ambient lighting interference during image acquisition although most of this effect was corrected

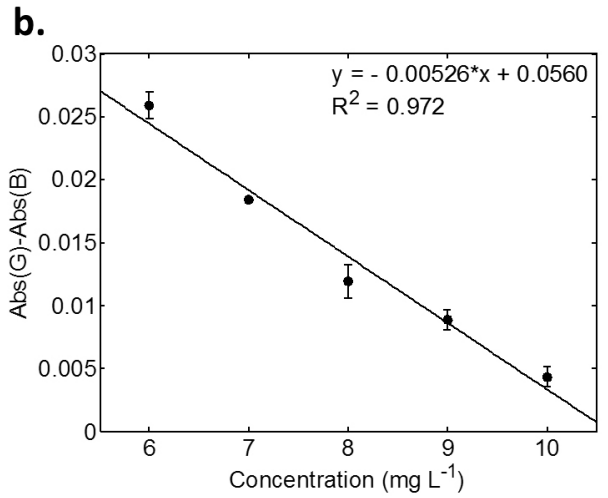

Figure 3. Effect of AA (6.0-10.0 $\left.\mathrm{mg} \mathrm{L}^{-1}\right)$ in the formation of quinoneimine chromophore resulting from the reaction of $20 \mu \mathrm{L}$ of glucose solution (10.0 mg $\left.\mathrm{L}^{-1}\right)$ in $2 \mathrm{~mL}$ of color reagent: (a) calibration curve obtained from UV-Vis data; (b) calibration curve using image data, where Abs(G) is the resolved absorbance for $\mathrm{G}$ channel and $\mathrm{Abs}(\mathrm{B})$ is the resolved absorbance for B channel. Vertical bars represent the standard deviation obtained in a triplicate of each point. 
Table 2. Nominal and predicted concentrations for UV-Vis and imaging method. Average relative error in percentage and $t$-value calculated by Student's $t$-test are shown

\begin{tabular}{lccc}
\hline & & Brand & C \\
\cline { 2 - 4 } Method & A & B & \\
\hline UV-Vis & 8.0 & 8.0 & 8.0 \\
\hline Nominal concentration / (mg L-1) & $8.0 \pm 0.3$ & $8.0 \pm 0.2$ & $7.9 \pm 0.2$ \\
Predicted concentration / (mg L & & 1.25 \\
Relative error / \% & 0.01 & 0.04 & -0.87 \\
$t$-Value & 0.01 & 0.03 & 8.0 \\
\hline Imaging & & & $8.3 \pm 0.6$ \\
Nominal concentration / (mg L-1) & 8.0 & $8.1 \pm 0.6$ & 3.75 \\
Predicted concentration / (mg L & 1.25 & 0.87 \\
Relative error / \% & $8.2 \pm 0.5$ & 0.29 & \\
$t$-Value & 2.50 & & \\
\hline
\end{tabular}

during absorbance calculation. A Student's $t$-test was applied to the samples' triplicate and the calculated $t$-value is also shown in Table 2. All calculated $t$-values for imaging method were smaller than the critical $t$-value $(4.3, p$-value $=0.975)$, therefore showing no statistical difference at a $95 \%$ confidence level between the predicted concentrations of AA calculated by the imaging method and the nominal concentrations for the three brands analyzed.

The precision of imaging method was evaluated by relative standard deviation (RSD). For brands A, B and C the RSD were equal to $6.1,7.4$ and $7.2 \%$, respectively. Using UV-Vis spectroscopy, the RSD were smaller due to its better sensitivity: $3.7 \%$ (brand A), 2.5\% (brand B), and $2.5 \%$ (brand C). Although the RSD is higher in imaging method, the values obtained $(<10 \%)$ seems to be adequate to this type of analysis, since the speed and portability of this technique competes with its sensitivity. The accuracy of imaging method was evaluated by its recovery. Recovery ranging from $96.2-108.7 \%$ was found for brand A; 93.7-108.7\% for brand B; and 96.2-111.2\% for brand C. Using UV-Vis spectroscopy, the recoveries range were shorter: $96.2-103.7 \%$ (brand A); $97.5-102.5 \%$ (brand B); 96.2-101.2\% (brand C). These recovery values are close to $100 \%$, proving the capability of both curves to predicted concentrations of AA with low error.
The limit of detection (LOD) and quantification (LOQ) were estimated for the image method using the modern IUPAC recommendation, ${ }^{25}$ and they were equal to 0.055 and $0.166 \mathrm{mg} \mathrm{L}^{-1}$, respectively. Using UV-Vis spectroscopy, the LOD and LOQ values were equal to 0.045 and $0.138 \mathrm{mg} \mathrm{L}^{-1}$, respectively. Although the LOD and LOQ values calculated for the image method are slightly elevated, they do not affect the analysis of commercial vitamin C samples, since its content in solution is much higher. The AA content calculated by the image method in commercial vitamin $\mathrm{C}$ samples is summarized in Table 3 .

In literature, there are only a few works reporting the quantification of AA from digital image analysis, however, these methodologies are mainly based on the use of noble metal-based nanoparticles and click chemistry reactions, which require specific and expensive reagents, in addition to relatively laborious and time-consuming procedures. ${ }^{17-20}$ In this context, we can attest that the results herein are very interesting due to three main facts: $(i)$ we have worked with both standard and real commercial samples of AA, with the latter being a more complex chemical composition; (ii) we made use of commercially available and very low cost reagent (\$15.00USD/250 mL); (iii) our analyses were performed using a very portable image acquisition device (smartphone); and (iv) the method requires little handling and only about fifteen minutes to be completed.

Table 3. Reference and predicted mass content of AA using UV-Vis spectroscopy and image method for samples of brand A, B, and C

\begin{tabular}{lccccc}
\hline Brand & $\begin{array}{c}\text { Reference labeled } \\
\text { content of ascorbic acid }\end{array}$ & $\begin{array}{c}\text { Predicted content } \\
\text { by UV-Vis }\end{array}$ & $\begin{array}{c}\text { Relative error by } \\
\text { UV-Vis / } \%\end{array}$ & $\begin{array}{c}\text { Predicted content } \\
\text { by image }\end{array}$ & $\begin{array}{c}\text { Relative error } \\
\text { by image / \% }\end{array}$ \\
\hline A & $1.0 \mathrm{~g}$ & $1.0 \pm 0.04 \mathrm{~g}$ & 0.01 & $1.0 \pm 0.1 \mathrm{~g}$ & 2.50 \\
$\mathrm{~B}$ & $500 \mathrm{mg}$ & $500 \pm 12 \mathrm{mg}$ & 0.04 & $506 \pm 37 \mathrm{mg}$ & 1.20 \\
$\mathrm{C}$ & $1.0 \mathrm{~g}$ & $1.0 \pm 0.03 \mathrm{~g}$ & 1.25 & $1.0 \pm 0.1 \mathrm{~g}$ & 3.75 \\
\hline
\end{tabular}




\section{Conclusions}

We have reported herein a new methodology for determining AA in solution based on the analysis of digital images obtained with a smartphone. The principle of this proposed strategy is based on a lower quantity of chromophore quinoneimine (resulting from the ECM approach for determining glucose) being formed as the AA concentration in media is increased. One of the main goals of this work was to explore a well established adverse effect for the analysis of glucose and successfully apply to the quantitative analysis of AA in aqueous media, especially from samples of vitamin $\mathrm{C}$ supplement in tablets. An image analysis approach was investigated in order to predict concentrations of AA in aqueous solution and the results found were very satisfactory for both calibration (standard AA) and prediction (commercial vitamin C supplements) sets. Therefore, this method can be highlighted due to its short analysis time, low cost, high availability of enzymatic colorimetric color reagent, and the simplicity and portability of the instrumentation being utilized (a smartphone). Furthermore, this new approach may contribute to the development and consolidation of a new field for chemical analysis, which includes quantitative evaluation of a broad spectrum of chemical analytes based on the use of mobile phones and image analysis.

\section{Acknowledgments}

The authors would like to acknowledge the structural and financial support from UFRN, CAPES and CNPq. M. S. C. would like to thank UFRN for the scholarship. C. L. M. M. and A. C. O. N. thank the Post-Graduate Program in Chemistry (PPGQ) of UFRN and CAPES. K. M. G. L. thanks the CNPq (305962/2014-0).

\section{References}

1. da Silva, L. C.; de Lima, D. F.; Silva, J. A.; de Morais, C. L. M.; Albuquerque, B. L.; Bortoluzzi, A. J.; Domingos, J. B.; Araújo, R. M.; Menezes, F. G.; de Lima, K. M. G.; J. Braz. Chem. Soc. 2016, 26, 1067.

2. Hemmateenejad, B.; Akhond, M.; Mohammadpour, Z.; Mobaraki, N.; Anal. Methods 2012, 4, 933.

3. Russ, J. C.; J. Food Sci. 2015, 80, 1974.
4. Yu, H.; Le, H. M.; Kaale, E.; Long, K. D.; Layloff, T.; Lumetta, S. S.; Cunningham, B. T.; J. Pharm. Biomed. Anal. 2016, 125, 85.

5. Martinez, A. W.; Phillips, S. T.; Carrilho, E.; Thomas, S. W.; Sindi, H.; Whitesides, G. M.; Anal. Chem. 2008, 80, 3699.

6. Zhu, H.; Sencan, I.; Wong, J.; Dimitrov, S.; Tseng, D.; Nagashima, K.; Ozcan, A.; Lab Chip 2013, 13, 1282.

7. Morais, C. L. M.; Neves, A. C. O.; Menezes, F. G.; Lima, K. M. G.; Anal. Methods 2016, 8, 6458.

8. Morais, C. L. M.; Lima, K. M. G.; Anal. Methods 2015, 7, 6904.

9. Morais, C. L. M.; Lima, K. M. G.; Talanta 2014, 126, 145.

10. Du, J.; Cullen, J. J.; Buettner, G. R.; Biochim. Biophys. Acta 2012, 1826, 443.

11. Klimczak, I.; Gliszczyńska-Świgło, A.; Food Chem. 2015, 175, 100.

12. Kemmegne-Mbouguen, J. C.; Angnes, L.; Sens. Actuators, B 2015, 212, 464.

13. Santos, D. A.; Lima, K. P.; Março, P. H.; Valderrama, P.; J. Braz. Chem. Soc. 2016, 27, 1912.

14. VanderJagt, D. J.; Garry, P. J.; Hunt, W. C.; Clin. Chem. 1986, 32,1004 .

15. Vermeir, S.; Hertog, M. L. A. T. M.; Schenk, A.; Beullens, K.; Nicolaï, B. M.; Lammertyn, J.; Anal. Chim. Acta 2008, 618, 94.

16. Wu, X.; Diao, Y.; Sun, C.; Yang, J.; Wang, Y.; Sun, S.; Talanta 2003, 59, 95.

17. Zhang, Y.; Li, B.; Xu, C.; Analyst 2010, 135, 1579.

18. Hemmateenejad, B.; Shakerizadeh-Shirazi, F.; Heidari, S.; Shahrivar-kevishahi, A.; Anal. Methods 2015, 7, 6318.

19. Gomes, M. S.; Trevizan, L. C.; Nóbrega, J. A.; Kamogawa, M. Y.; Quim. Nova 2008, 31, 1577.

20. Ferreira, D. C. M.; Giordano, G. F.; Soares, C. C. D. S. P.; de Oliveira, J. F. A.; Mendes, R. K.; Piazzetta, M. H.; Gobbi, A. L.; Cardoso, M. B.; Talanta 2015, 141, 188.

21. Menezes, F. G.; Neves, A. C. O.; de Lima, D. F.; Lourenço, S. D.; da Silva, L. C.; de Lima, K. M. G.; Quim. Nova 2015, 38,588 .

22. Jansson, P. J.; Jung, H. Y. E. R.; Lindqvist, C.; Nordstro, T.; Free Radical Res. 2004, 38, 855.

23. https://www.gimp.org/, accessed in May 2017.

24. Christodouleas, D. C.; Nemiroski, A.; Kumar, A. A.; Whitesides, G. M.; Anal. Chem. 2015, 87, 9170.

25. Olivieri, A. C.; Anal. Chim. Acta 2015, 868, 10.

26. Danzer, K.; Currie, L. A.; Pure Appl. Chem. 1998, 70, 993.

Submitted: February 13, 2017

Published online: May 17, 2017 